\title{
Spontaneous mode-selection in the self-propelled motion of a solid/liquid composite driven by interfacial instability.
}

\section{AUTHOR(S):}

Takabatake, Fumi; Magome, Nobuyuki; Ichikawa, Masatoshi; Yoshikawa, Kenichi

\section{CITATION:}

Takabatake, Fumi ... [et al]. Spontaneous mode-selection in the self-propelled motion of a solid/liquid composite driven by interfacial instability.. The Journal of chemical physics 2011, 134(11): 114704.

\section{ISSUE DATE:}

2011-03-21

\section{URL:}

http://hdl.handle.net/2433/139584

\section{RIGHT:}

(C) 2011 American Institute of Physics 


\title{
Spontaneous mode-selection in the self-propelled motion of a solid/liquid composite driven by interfacial instability
}

\author{
Fumi Takabatake, ${ }^{1}$ Nobuyuki Magome, ${ }^{2}$ Masatoshi Ichikawa, ${ }^{1}$ and Kenichi Yoshikawa ${ }^{1, a)}$ \\ ${ }^{1}$ Department of Physics, Graduate School of Science, Kyoto University, Kyoto 606-8502, Japan \\ ${ }^{2}$ Institute for Integrated Cell-Material Sciences (iCeMS), Kyoto University, Kyoto 606-8501, Japan
}

(Received 30 September 2010; accepted 22 February 2011; published online 17 March 2011)

\begin{abstract}
Spontaneous motion of a solid/liquid composite induced by a chemical Marangoni effect, where an oil droplet attached to a solid soap is placed on a water phase, was investigated. The composite exhibits various characteristic motions, such as revolution (orbital motion) and translational motion. The results showed that the mode of this spontaneous motion switches with a change in the size of the solid scrap. The essential features of this mode-switching were reproduced by ordinary differential equations by considering nonlinear friction with proper symmetry. (c) 2011 American Institute of Physics. [doi:10.1063/1.3567096]
\end{abstract}

\section{INTRODUCTION}

Thermal engines transfer chemical energy into mechanical energy through the generation of heat and obey the second law of thermodynamics: the upper limit of thermal efficiency is determined by the difference between the high and low temperatures. On the other hand, living organisms extract kinetic energy from chemical energy directly with a high energy-conversion efficiency under isothermal conditions. Despite previous extensive studies on motor proteins, our understanding of the underlying mechanisms remains at a primitive stage. Recent studies on the single-molecule observation of motor proteins have revealed that biological macromolecules work under highly fluctuating conditions. ${ }^{1}$ Thus, it may be of scientific value to clarify the generation of regular motion in a macroscopic system with fluctuating elements.

The spontaneous motion of liquid droplets, solid particles, and gels under nonequilibrium conditions has been investigated both experimentally and theoretically. ${ }^{2-8}$ Among the phenomena of spontaneous motions, the self-agitation of a fluid interface is well known and has been studied for over a century, especially in the field of chemical engineering in terms of the chemical Marangoni effect. ${ }^{9-13}$ The chemical Marangoni effect is induced by local variations in interfacial tension, which are caused by a chemical concentration gradient under isothermal conditions. Recently, it was found that irregular interfacial agitation caused by the Marangoni instability can be transformed to regular motion by choosing a suitable physical or chemical boundary condition. ${ }^{14-17}$

In the present study, we found experimentally that a solid/liquid composite exhibits regular motion under spatially isotropic boundary conditions by breaking its anteriorposterior symmetry. The results showed that a composite driven by the chemical Marangoni effect assumes a certain mode of spontaneous motion depending on the relative size of the solid with respect to that of the liquid.

\footnotetext{
a) Author to whom correspondence should be addressed. Electronic mail: yoshikaw@ @sphys.kyoto-u.ac.jp. FAX: +81-75-753-3779.
}

\section{EXPERIMENTAL}

A droplet of oleic acid and a solid sodium oleate (soap) were floated on water in a petri dish as shown in Fig. 1(a). Water was purified with a a MilliQ filtering system (Millipore). The volume of the oil droplet was $100 \mu$ l. Oleic acid (analytical grade) was obtained from Wako Pure Chemical Industries, Japan. A solid column of sodium oleate with a desired axial length was chosen from a commercial sample (Code 257-02) obtained from Nacalai Tesque [Fig. 1(b)]. The movement of the solid/liquid composite was captured by a digital video camera at 30 frames per second at room temperature, and then analyzed by an image-processing software (ImageJ). Example of the video frames is given in Fig. 1(c).

\section{RESULTS AND DISCUSSIONS}

Figure 2 exemplifies the characteristic modes of spontaneous motion of a solid/liquid composite on the water phase: (left) shadow graph images of the solid/liquid composite and (right) trajectory of the droplet-center over $5 \mathrm{~s}$. The solid/liquid composite shows continuous motion by taking the solid column as the front side over a period of several minutes. Investigation on the moving composite indicates the occurrence of milky stream of surfactant micelles on the rear side opposite to the solid column, indicating the occurrence of eliminating or dispelling flow of the surfactant from the composite. When the axial length of the solid column is ca. $1 \mathrm{~mm}$, the composite exhibits spinning motion, as shown in Fig. 2(a). When the axial length is ca. $3 \mathrm{~mm}$, the composite exhibits translational motion accompanied by relatively slow spinning, as shown in Fig. 2(b). When the axial length is ca. $5 \mathrm{~mm}$, the composite exhibits revolution (orbital motion) together with the spinning motion around its centroid [Fig. 2(c)], where the period of revolution is equal to that of the spinning motion.

The panels on the left in Figs. 3 I(a)-(c) show time traces of the angular velocity $\omega$ and the speed $|\boldsymbol{v}|$ of motion over $30 \mathrm{~s}$. The panels on the right show histograms of the angular velocity $\omega$ over $60 \mathrm{~s}$. The direction of spinning motion spontaneously switches in a stochastic manner, as shown in 
(a)

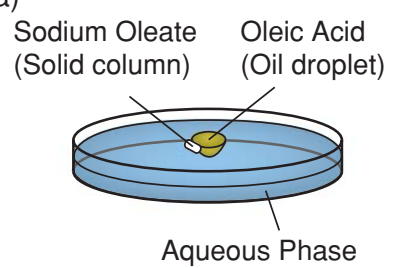

(c)
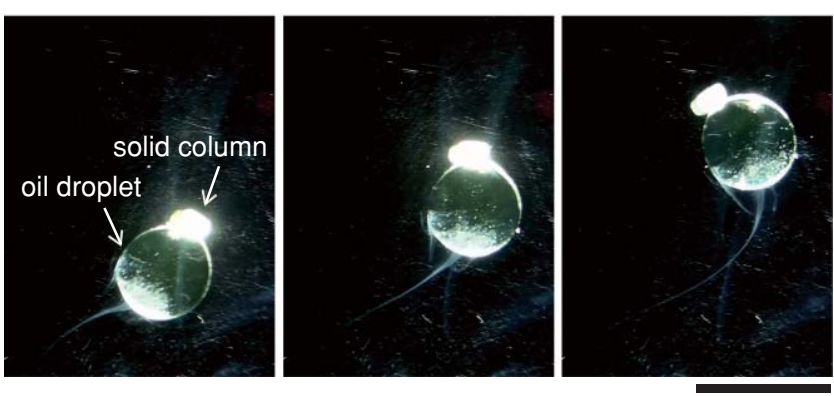

$10 \mathrm{~mm}$

FIG. 1. Experimental system. (a) Overall set-up. (b) Morphology of the solid column, where the axial length is changed between 1 and $6 \mathrm{~mm}$. (c) Darkfield optical observation on a self-propelled solid/liquid composite. The axial length of the solid column is ca. $3 \mathrm{~mm}$. Time interval is $1 \mathrm{~s}$.

Fig. $3 \mathrm{I}(\mathrm{a})$, where $l \simeq 1 \mathrm{~mm}$. The probability of the angular velocity shows a pair of symmetric peaks in addition to the lower peak of $\omega \simeq 0$, which reflects the fluctuation of the direction of spinning. When the solid becomes larger, $l \simeq 3 \mathrm{~mm}$, a distribution with a broad peak appears at around $\omega \simeq 0$, as shown in Fig. $3 \mathrm{I}(\mathrm{b})$, indicating that the composite exhibits mostly translational motion accompanied by fluctuation of its orientation. When the column size is around $5 \mathrm{~mm}$, as shown in Fig. $3 \mathrm{I}(\mathrm{c}-1, \mathrm{c}-2)$, the peak of the director deviates from $\omega=0$, which corresponds to the occurrence of the orbital motion [see Fig. 2(c)]. In Fig. 3 I(c-1) is exemplified the generation of the right-handed and left-handed motions. During the observation on the similar experimental condition, either mode with the right-left symmetry breaking is encountered as in Fig. $3 \mathrm{I}(\mathrm{c}-2)$. Thus, the mode-switching of this selfmotion depending on the size $l$ of the solid column is represented by the characteristics, or symmetry, of the probability distribution with $\omega$. Summarizing the results, a phase diagram of the mode of spontaneous motion as a function of the axial length $l$ is given in Fig. 3 II. As indicated by the tilted broken lines, there exists a finite width of the coexistence region between the different modes, suggesting the bistability on the system characteristics.

In contrast to the self-motion exemplified in Figs. 2 and 3 when a droplet of oleic acid is placed on a water surface without solid soap, no self-propelled motion is generated. Instead, the solid scrap of sodium oleate exhibits self-motion for only 1-2 s and then stops. This short life-time of spontaneous motion of the scrap of sodium oleate is explained as follows. When a scrap is placed on water, sodium oleate molecules tend to spread out at the water/air interface because of its characteristics as a surfactant. This kinetic process causes temporal instability at the interface near the scrap and drives the scrap for a short time. After 1-2 s, the interface is covered

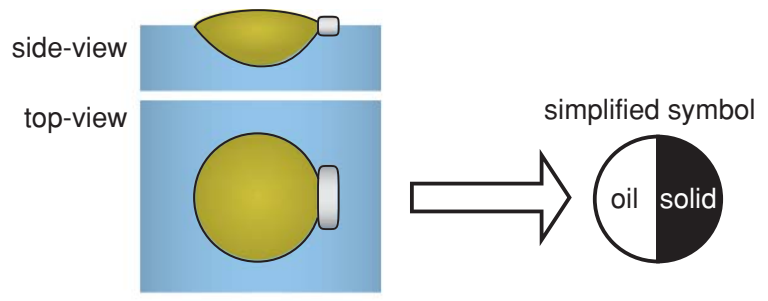

(a)
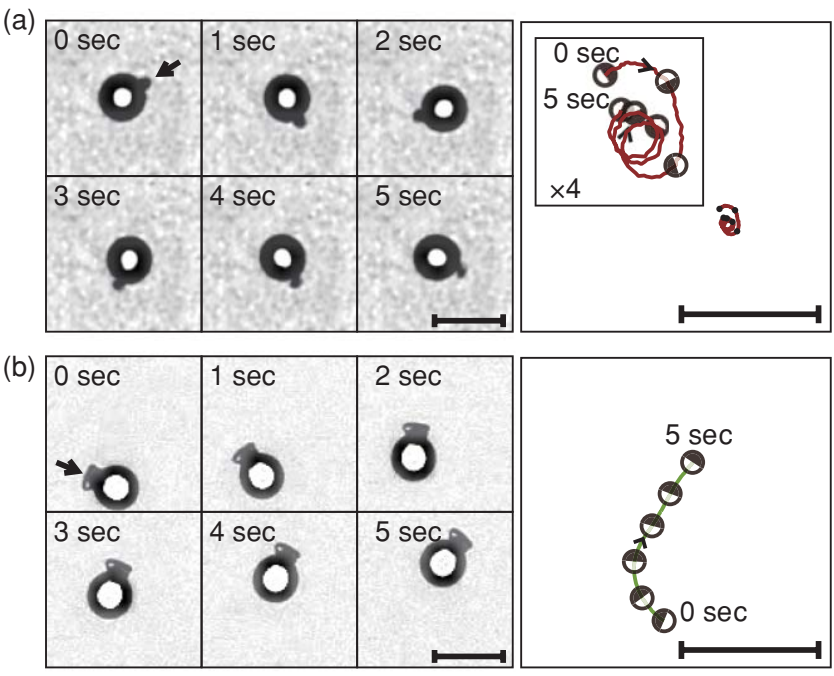

(c)

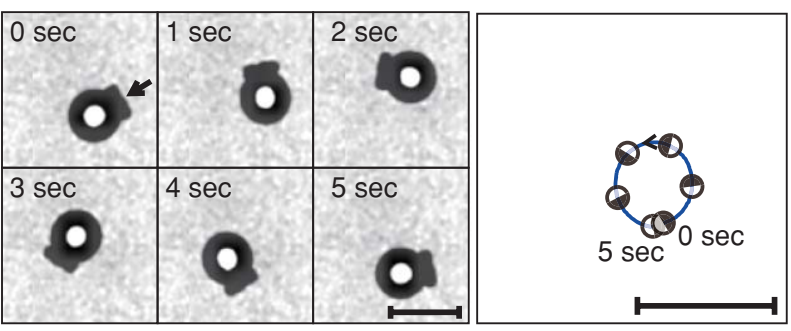

FIG. 2. Examples of the characteristic spontaneous motion of a solid/liquid composite depending on the size of the solid, where the volume of the droplet was kept constant at $100 \mu \mathrm{l}$. The upper panels show schematic images of the composite together with the corresponding simplified symbol (symbolic representation). (a, b, c) Left: Shadow graph images of the composite with the backlight, where the circular object is the liquid droplet. The white inner circle of the droplet is an artifact due to the reflection image (time interval: 1 $\mathrm{s}$, scale bar: $10 \mathrm{~mm}$ ). The arrows indicate the position of the solid column. Right: Trajectory of the center of the droplet (scale bar: $10 \mathrm{~mm}$ ). (a) The axial length of the solid column, $l \simeq 1 \mathrm{~mm}$. The solid column exhibits rotational motion around the periphery of the droplet. (b) $l \simeq 3 \mathrm{~mm}$. Translational motion is observed accompanied by slow rotation. (c) $l \simeq 5 \mathrm{~mm}$. Revolution is generated together with rotational motion around its centroid with the same periodicity.

with a surfactant monolayer, which results in the termination of spontaneous motion.

Next, let us discuss the cause of the self-motion of a solid/liquid composite, in terms of surface instability. The $\mathrm{oil} /$ water interfacial tension near the solid is lowered because of the formation of a surfactant monolayer dissolved from the solid column. On the other hand, the interfacial tension far from the solid column remains high because of the acidic nature of the oil, oleic acid. Thus, it is expected that a spatial gradient of interfacial tension is generated around the composite, where the tension near the solid column is low and that far from the solid column is high. As is exemplified in Fig. 1(c), the surfactant molecules (oleate molecules) existing around the oil/water interface tend to escape toward 
I (a) Spin
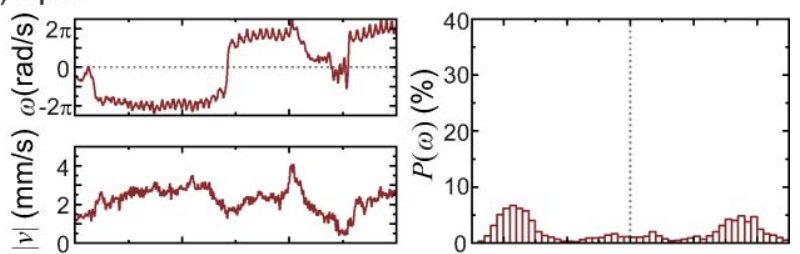

(b) Translation
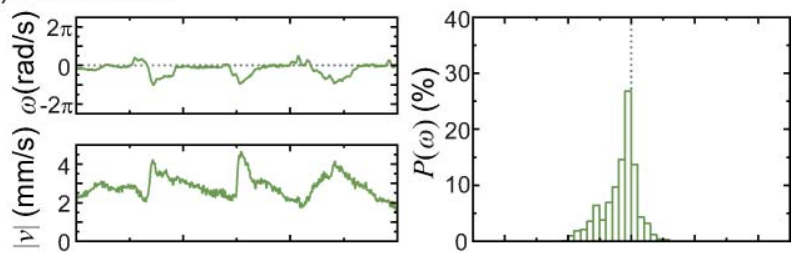

(c-1) Orbital motion
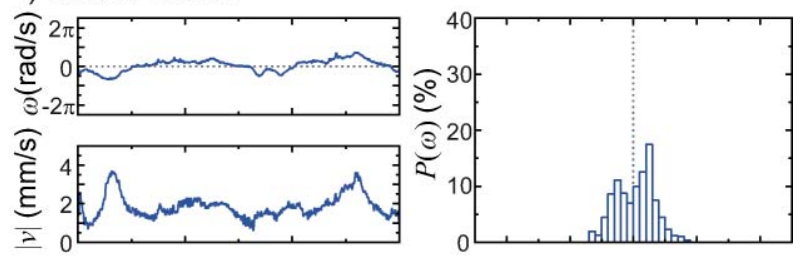

(c-2) Orbital motion
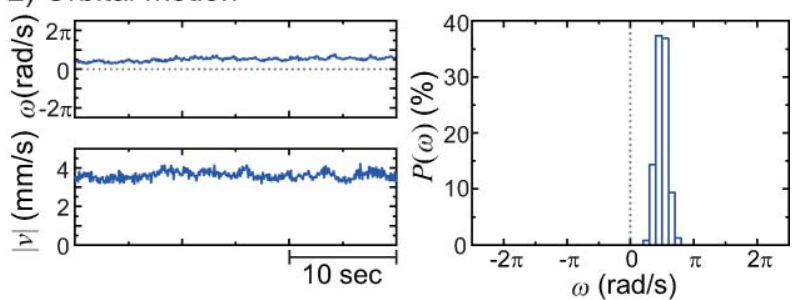

II

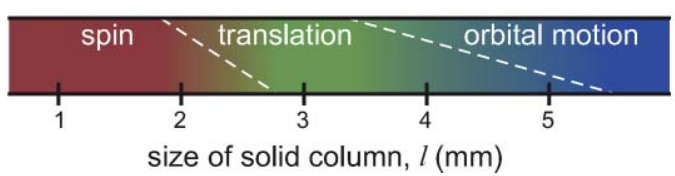

FIG. 3. Experimental characteristics of spontaneous motion. I: Analysis of the motion. (a), (b), and (c-2) are the same observations as in Figs. 2(a), 2(b) and 2(c), respectively. Left: Time traces of the angular velocity $\omega$ and the speed $|\boldsymbol{v}|$ of motion. Right: Histograms of the angular velocity $\omega$ over 60 s. (a) The axial length of the solid column, $l \simeq 1 \mathrm{~mm}$. (b) $l \simeq 3 \mathrm{~mm}$. (c-1, c-2) $l \simeq 5 \mathrm{~mm}$. For (c-1) and (c-2), in order to indicate the characteristic of bimodality on the orbital motion, different time-stage is given. II: Phase diagram of spontaneous motion, showing the dependence of the motional modes on the axis length of the solid column.

the bulk aqueous solution by making an eliminating flow from the rear side. Due to such dissipative flow of the surfactant, we can consider that tangential stress is maintained around the oil/water surface because of the gradient in the surfactant concentration at the interface, or the surface tension. In response to this stress, as depicted schematically in Fig. 4, shear flow is induced around the composite. Thus, it is highly probable that the driving force, which propels the droplet by taking the solid as the forward side, is caused by the momentum transfer through the generation of shear flow. ${ }^{18,19}$ On the other hand, convection inside the droplet has almost no effect on the driving force for the translational motion, due to the nature of the incompressible fluid, i.e., $\operatorname{div} \boldsymbol{u} \simeq 0$, where $\boldsymbol{u}$ is the flow inside the oil droplet.

Based on these considerations, the mechanism of persistent motion is described as follows. The surfactant molecules

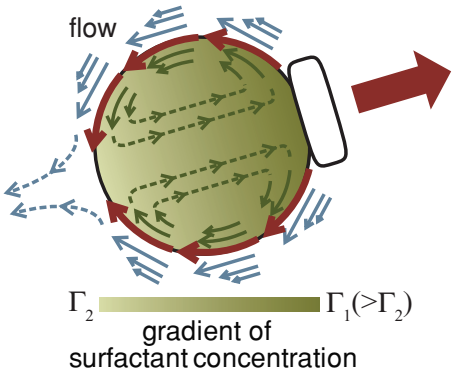

FIG. 4. Schematic illustration of the lines of flow induced by the tangential stresses (Marangoni stresses).

at the oil/water boundary are released to the water phase from the backside of the droplet. As the result of competition between the surfactant-supply from the solid column and escape into the bulk solution, the spatial gradient of the surface tension is maintained. Through such dissipative mechanism, the composite continues self-propelled motion for a relatively long period (more than several minutes).

As described above, the gradient of the interfacial tension induces the Marangoni convection around the composite and exerts the propulsion force on the composite by the momentum transfer through the occurrence of shear flow parallel to the interface, as schematically shown in Fig. 4. The propulsion force acting on the composite may be simulated by solving the Stokes equation, taking account of the gradient of the oil/water interfacial tension, ${ }^{18}$ as a subject for future study. However, in order to grasp the essence on the bifurcation of self-propelled motion, here we would like to adopt a simple theoretical model that the translational motion of the composite is caused by the apparent driving force $\boldsymbol{F}$, of which the magnitude $|\boldsymbol{F}|$ is time-independent and the orientation exhibits time-dependence. Under such framework of the modeling, translational motion is expressed as

$$
\begin{gathered}
\frac{\mathrm{d} \boldsymbol{r}}{\mathrm{d} t}=\boldsymbol{v} ; m \frac{\mathrm{d} \boldsymbol{v}}{\mathrm{d} t}=-\eta_{v} \boldsymbol{v}+\boldsymbol{F} \\
\text { with } \quad \boldsymbol{F}=\left(\begin{array}{c}
F_{x} \\
F_{y}
\end{array}\right)=\left(\begin{array}{c}
F \cos \theta \\
F \sin \theta
\end{array}\right), \quad F=|\boldsymbol{F}|,
\end{gathered}
$$

where $m$ is the mass of the composite, $\boldsymbol{r}$ is the displacement of the center of mass, $\boldsymbol{v}$ is the velocity of translational motion, and $\eta_{v}>0$ is the friction coefficient. $\boldsymbol{F}$ is the driving force due to the above-mentioned Marangoni stresses. In Eq. (2), $\theta$ is the angle of the axis that combines the centers of mass of the oil droplet and the solid scrap, as shown schematically in Fig. 5.

As for the rotational motion, we adopt a term of nonlinear friction as follows:

$$
\frac{\mathrm{d} \theta}{\mathrm{d} t}=\omega ; I \frac{\mathrm{d} \omega}{\mathrm{d} t}=-\eta_{\omega}(\omega) \omega+\xi_{\omega}(t)
$$

where $\omega$ is the angular velocity and $I$ is the moment of inertia. $\eta_{\omega}(\omega)$ is the friction function that includes the contribution from the driving torque in addition to the usual positive friction. $\xi_{\omega}$ represents the random fluctuation due to the interfacial and fluidic instability. To interpret the essential features of mode-switching, we simply adopt a symmetry requirement: 
$\eta_{\omega}(\omega)=\eta_{\omega}(-\omega)$. As shown in the picture of Fig. 1(c), together with the experimental data of Fig. 3, the composite tends to cause rotational motion, accompanied by the spontaneous symmetry breaking. In order to take into account of such self-driving force on the rotation, we adopt negative rotational "friction," $-\eta_{2} \omega^{2}<0$. So as to prevent the diverge of the rotation due to the negative friction, we also add the quartic term, $\eta_{3} \omega^{4}>0$. Thus, the friction function $\eta_{\omega}(\omega)$, including not only the frictional force but also the driving force due to Marangoni instability ${ }^{10}$ together with geometrical asymmetry, can be described as

$$
\eta_{\omega}(\omega)=\eta_{1}-\eta_{2} \omega^{2}+\eta_{3} \omega^{4}
$$

with positive coefficients $\eta_{1}, \eta_{2}$, and $\eta_{3}$.

By nondimensionalizing procedure, the motion of the solid/liquid composite is transformed into the following equations:

$$
\begin{gathered}
\frac{\mathrm{d} \tilde{x}}{\mathrm{~d} \tilde{t}}=\tilde{v}_{x} ; \frac{\mathrm{d} \tilde{v}_{x}}{\mathrm{~d} \tilde{t}}=-\tilde{v}_{x}+\tilde{F} \cos \theta, \\
\frac{\mathrm{d} \tilde{y}}{\mathrm{~d} \tilde{t}}=\tilde{v}_{y} ; \frac{\mathrm{d} \tilde{v}_{y}}{\mathrm{~d} \tilde{t}}=-\tilde{v}_{y}+\tilde{F} \sin \theta, \\
\frac{\mathrm{d} \theta}{\mathrm{d} \tilde{t}}=\tilde{\omega} ; \frac{\mathrm{d} \tilde{\omega}}{\mathrm{d} \tilde{t}}=-\left(\tilde{\eta}_{1}-\tilde{\eta}_{2} \tilde{\omega}^{2}+\tilde{\eta}_{3} \tilde{\omega}^{4}\right) \tilde{\omega}+\tilde{\xi}_{\omega},
\end{gathered}
$$

where $t=\left(m / \eta_{v}\right) \tilde{t}$ and $\boldsymbol{r}=\sqrt{I / m} \tilde{\boldsymbol{r}}$. The variables in these equations, $\tilde{t}, \tilde{x}, \tilde{y}, \tilde{v}_{x}, \tilde{v}_{y}$, and $\tilde{\omega}$, are dimensionless. The dimensionless friction function $\tilde{\eta}_{\omega}$ is

$$
\tilde{\eta}_{\omega}(\tilde{\omega})=\tilde{\eta}_{1}-\tilde{\eta}_{2} \tilde{\omega}^{2}+\tilde{\eta}_{3} \tilde{\omega}^{4}=\tilde{\eta}_{1}\left(1-\alpha \tilde{\omega}^{2}+\beta \tilde{\omega}^{4}\right),
$$

where $\alpha=\tilde{\eta}_{2} / \tilde{\eta}_{1}$ and $\beta=\tilde{\eta}_{3} / \tilde{\eta}_{1}$. In Eq. (8), the friction function becomes negative within a certain range of angular velocity $\omega$ if $\alpha^{2} / \beta>4$, which implies that the active force is generated. Additionally, it is noted that the fixed points of Eq. (7) at $\tilde{\omega}^{*} \neq 0$ become more stable than a stable fixed point at $\tilde{\omega}^{*}=0$ when $\alpha^{2} / \beta>16 / 3$.

Figure 5 exemplifies the numerical results based on Eqs. (5)-(7). In numerical calculations, $\tilde{\xi}_{\omega}$ is simply taken as the white Gaussian noise whose dispersion is $s$. It is to be noted that the essential features of the experimental results as shown in Fig. 3 are reproduced by choosing appropriate parameters. In these simulations, to gain an insight on the mechanism of the mode bifurcation, we examine the effect of two parameters, $\alpha$ and $s$, while others are fixed to be constants.

A comparison between Figs. 5(a) and 5(b) reveals modeswitching from rotational motion to translational motion, corresponding the experiments in (a) and (b) of Fig. 2. The larger value of $\alpha^{2} / \beta$ in (a) with respect to (b) in Fig. 5 is regarded as to represent a higher possibility of symmetry-breaking between clockwise and anticlockwise spinning motions under irregular fluctuation due to the interfacial instability. This matches with the experimental trend that small scrap tends to rotate faster. Since $m$ is fixed in the equations, the size change of the solid column is described as the relative value of mass of the solid column with that of the droplet, which affects $\sqrt{I / m}$, a coefficient of the length scale. Figure 5 is plotted in $\boldsymbol{r}$ space, i.e., each mass of the solid column proportional to the length $l$ in the experiment is used for the magnitude of the scale in the corresponding plot as modulus $\boldsymbol{r} \sim \tilde{\boldsymbol{r}} \sqrt{l}$

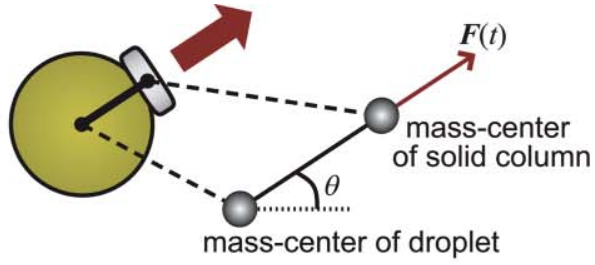

(a)

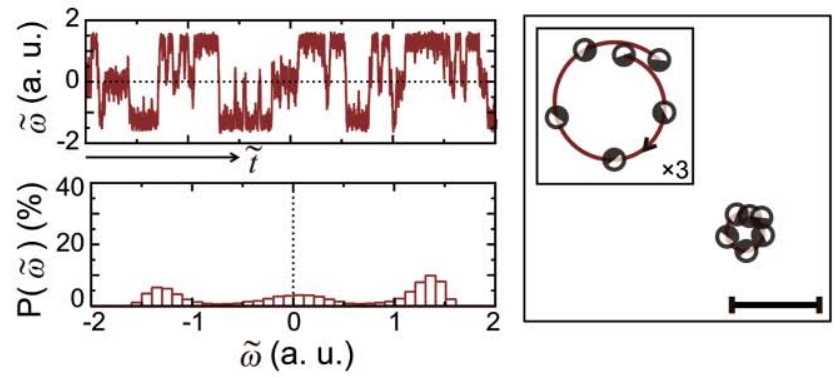

(b)
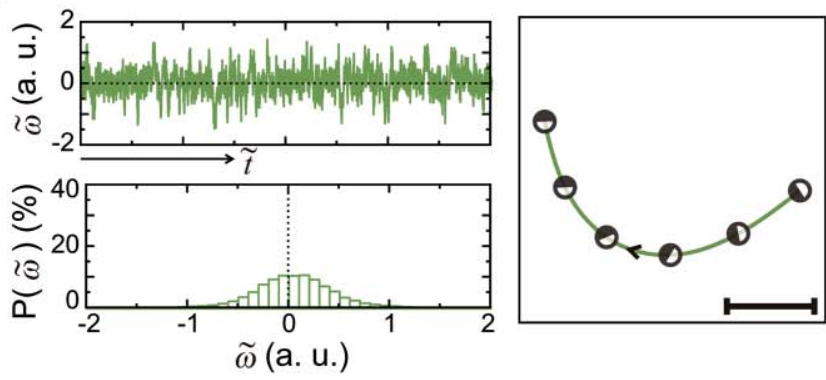

(c-1)

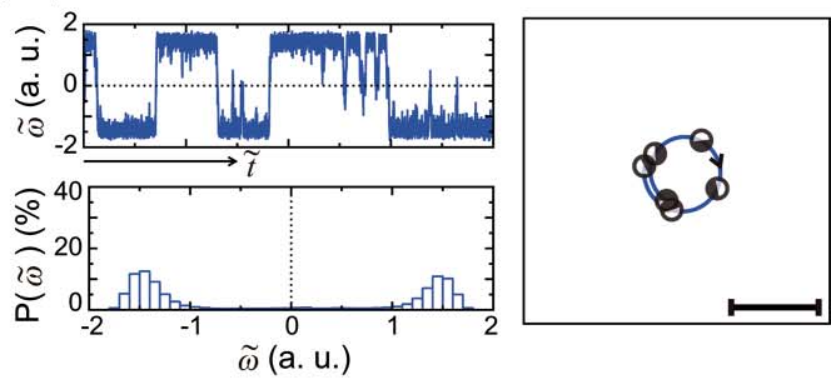

(c-2)
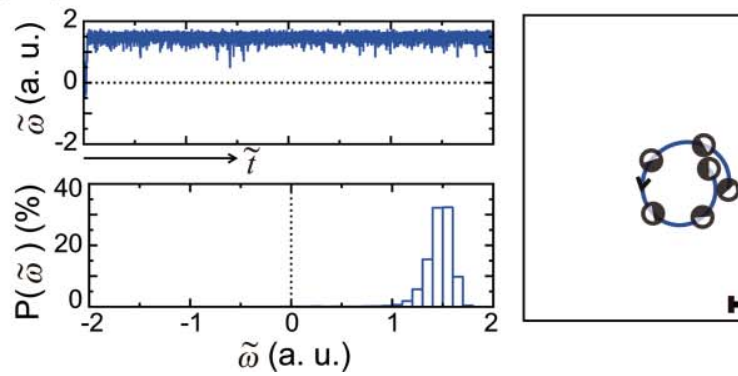

FIG. 5. Numerical simulation based on Eqs. (5)-(7). The top panel shows a frame format of the model. Left: Time traces of the angular velocity $\tilde{\omega}$ and histograms of $\tilde{\omega}(0 \leq \tilde{t} \leq 1000)$. Right: Trajectory of the centroid of the composite in $\boldsymbol{r}$ space for a short period $(400 \leq \tilde{t} \leq 405)$. The scales are the same on the four trajectories except for the inset. (a) $\alpha=2.4, s=0.3$. (b) $\alpha=1.8, s=0.3$. (c-1) $\alpha=2.7, s=0.5$. (c-2) $\alpha=2.7, s=0.3$. The other fixed parameters are $\tilde{\eta}_{1}=1, \beta=1, \tilde{F}=0.1$. The initial values are $\tilde{x}(0)=\tilde{y}(0)=0, \tilde{v}_{x}(0)=\tilde{v}_{y}(0)=0, \theta(0)=0$, and $\tilde{\omega}(0)=0$. 
[in (a) $l=1$, in (b) $l=3$, and in (c) $l=5$ ]. Thus, motions in Figs. 5 (c-1, c-2) are regarded as rotation with larger orbital radii than that in (a). The larger value of $s$ in (c-1) represents approximately a high possibility that the composite is subject to larger motional fluctuation due to the instability or external perturbations. This large fluctuation causes the flip-flop fluctuation of the direction of the orbital motion. When the sizes of the solid column and the droplet deviate largely from the similar magnitude, rotational motion of the composite shows bistability of clockwise and anticlockwise as in the experiments. Therefore, large enough unbalance between the sizes of the solid and the liquid in the composite, as in (a) and (c), corresponds to the state with large $\alpha^{2} / \beta$, and the balanced condition as (b) is described as the effect of small $\alpha^{2} / \beta$.

\section{CONCLUSION}

We have experimentally demonstrated the appearance of mode-switching in the self-propelled motion of a solid/liquid composite. The driving force is the interfacial instability caused by nonuniformity in the chemical concentration or by spontaneous symmetry breaking the geometric configuration on the composite. Mode-switching, among spinning, translational motion, and orbital motion, is caused by the changes in size of the solid column. The essential features of the experimental observation have been reproduced by adopting a simple model with ordinary differential equations, by taking account of the scheme of spontaneous symmetry breaking. Since almost all self-moving organelles and cells exhibit a head-tail asymmetry, our simple experimental and numerical models may give deeper insight into mode-switching in living systems.

\section{ACKNOWLEDGMENTS}

We gratefully acknowledge the assistance of Professor K. I. Agladze. We also thank Dr. K. H. Nagai, Dr. Y. Sumino,
Dr. N. Yoshinaga, and Dr. H. Kitahata for their helpful discussions and advice. This work was supported by a Sasagawa Scientific Research (Grant No. 22-220) from the Japan Science Society, by an iCeMS Cross-Disciplinary Research Promotion Project (OAP3-9), and by a Grantin-Aid for Challenging Exploratory Research (Grant No. 22654046) from the Japan Society for the Promotion of Science.

${ }^{1}$ M. Mickler, E. Schleiff, and T. Hugel, ChemPhysChem 9, 1503 (2008).

${ }^{2}$ U. Erdmann, W. Ebeling, L. Schimansky-Geier, and F. Schweitzer, Eur. Phys. J. B 15, 105 (2000).

${ }^{3}$ P. Tietro, R. Golestanian, I. Pagonabarraga, and F. Sagués, Phys. Rev. Lett. 101, 218304 (2008).

${ }^{4}$ J. Tersoff, D. E. Jesson, and W. X. Tang, Science 324, 236 (2009).

${ }^{5}$ S. J. Ebbens and J. R. Howse, Soft Matter 6, 726 (2010).

${ }^{6}$ N. Bassik, B. T. Abebe, and D. H. Gracias, Langmuir 24, 12158 (2008).

${ }^{7}$ M. Nagayama, S. Nakata, Y. Doi, and Y. Hayashima, Physica D 194, 151 (2004).

${ }^{8}$ T. Ohkuma and T. Ohta, Chaos 20, 023101 (2010).

${ }^{9}$ L. E. Scriven and C. V. Sternling, Nature (London) 187, 186 (1960).

${ }^{10}$ S. Nakata and S. Hiromatsu, Chem. Phys. Lett. 405, 39 (2005).

${ }^{11}$ T. Toyota, N. Maru, M. M. Hanczyc, T. Ikegami, and T. Sugawara, J. Am. Chem. Soc. 131, 5012 (2009).

${ }^{12}$ K. Nagai, Y. Sumino, H. Kitahata, and K. Yoshikawa, Phys. Rev. E 71, 065301 (2005).

${ }^{13}$ H. Kang, D. S. Perlmutter, V. B. Shenoy, and J. X. Tang, Biophys. J. 99, 2793 (2010).

${ }^{14} \mathrm{~F}$. Brochard, Langmuir 5, 432 (1989).

${ }^{15}$ Y. Sumino and K. Yoshikawa, Chaos 18, 026106 (2008).

${ }^{16}$ D. Quéré and A. Ajdari, Nature Mater. 5, 429 (2006).

${ }^{17}$ P. Reimann, Phys. Rep. 361, 57 (2002).

${ }^{18}$ N. O. Young, J. S. Goldstein, and M. J. Block, J. Fluid Mech. 6, 350 (1959).

${ }^{19}$ A. Diguet, R.-M. Guillermic, N. Magome, A. Saint-Jalmes, Y. Chen, K. Yoshikawa, and D. Baigl, Angew. Chem., Int. Ed. 48, 9281 (2009). 